\title{
Adjuvant apatinib treatment after resection of hepatocellular carcinoma with portal vein tumor thrombosis: a phase II trial
}

\author{
Hui-Chuan Sun", Xiao-Dong Zhu\#, Jian Zhou, Qiang Gao, Ying-Hong Shi, Zhen-Bing Ding, \\ Cheng Huang, Shuang-Jian Qiu, Ning Ren, Guo-Ming Shi, Jian Sun, Qing-Hai Ye, Xiao-Wu Huang, \\ Xin-Rong Yang, Jia Fan
}

Department of Liver Surgery and Transplantation, Liver Cancer Institute and Zhongshan Hospital, Fudan University, Shanghai, China Contributions: (I) Conception and design: HC Sun, J Fan; (II) Administrative support: HC Sun, J Fan; (III) Provision of study materials or patients: HC Sun, J Zhou, Q Gao, YH Shi, ZB Ding, C Huang, SJ Qiu, N Ren, GM Shi, J Sun, QH Ye, XW Huang, XR Yang, J Fan; (IV) Collection and assembly of data: All authors; (V) Data analysis and interpretation: XD Zhu; (VI) Manuscript writing: All authors; (VII) Final approval of manuscript: All authors.

"These authors contributed equally to this work.

Correspondence to: Jia Fan. Department of Liver Surgery and Transplantation, Liver Cancer Institute and Zhongshan Hospital, Fudan University, Shanghai 200032, China. Email: fan.jia@zs-hospital.sh.cn.

Background: Survival after resection of hepatocellular carcinoma (HCC) with portal vein tumor thrombosis (PVTT) still remains poor. Apatinib, a vascular endothelial cell growth factor receptor 2 inhibitor, has been shown to be safe and effective in patients with advanced HCC, so in the present study its efficacy and safety in the adjuvant setting was explored.

Methods: In this single-center, open-label phase II trial, the patients received apatinib (500 mg/day) until they experienced disease recurrence or intolerable toxicity. The primary endpoint was recurrence-free survival (RFS); the secondary endpoints included overall survival (OS) and safety.

Results: From a total of 49 patients who were screened between August 2017 and December 2018, 30 study participants received apatinib. According to the Liver Cancer Study Group of Japan classification of PVTT, there were 7, 11, and 12 participants with $\mathrm{Vp} 1, \mathrm{Vp} 2$, and $\mathrm{Vp} 3$, respectively. The median duration of treatment was 4.8 months [interquartile range (IQR): 2.0-8.8], and the median dose of apatinib was $339.7 \mathrm{mg} /$ day (IQR: 267.7-500 mg/day). The median follow-up was 14.3 months (IQR: 12.3-19.3). The median RFS was 7.6 months [95\% confidence interval (CI): 5.7-9.5 months]. The 1-year RFS rate and the 1-year OS rate were $36.1 \%$ and $93.3 \%$, respectively. A total of $29(96.7 \%)$ patients experienced adverse events, and 14 (46.7\%) had grade 3 or 4 adverse events. No treatment-related deaths occurred.

Conclusions: Apatinib was well tolerated in patients after resection of HCC with PVTT. The median RFS in this group was improved compared with that previously reported.

Trial registration: No.: NCT03261791 (ClinicalTrials.gov).

Keywords: Apatinib; hepatocellular carcinoma (HCC); portal vein

Submitted Jul 31, 2020. Accepted for publication Oct 16, 2020.

doi: $10.21037 / \mathrm{atm}-20-6181$

View this article at: http://dx.doi.org/10.21037/atm-20-6181

\section{Introduction}

Liver cancer is the sixth most common cancer and the fourth leading cause of cancer-related death worldwide (1). Hepatocellular carcinoma (HCC) is the most common type of liver cancer (75-85\%) (2), and the main treatment includes liver transplantation, liver resection, loco-regional therapy, and systemic therapy (2).

In China, $23 \%$ of patients with diagnosed HCC also have portal vein tumor thrombosis (PVTT) (3), which is an 
indicator of an advanced stage and a poor prognosis (4-6). Untreated patients with HCC and PVTT have a median survival of about 2.7-4.0 months. Chinese Liver Cancer Treatment Guidelines recommend surgical resection, systemic treatment, transarterial chemoembolization, and radiation therapy for the treatment of HCC with PVTT (7). On the other hand, the American Association for the Study of Liver Diseases only recommends systemic treatment for HCC patients with PVTT (8).

Surgical resection can improve the survival of selected patients with HCC and PVTT, but has been associated with a high incidence of tumor recurrence $(5,6)$. Total resection of liver cancer tissue and tumor thrombus is very challenging, especially for small tumor thrombus and micrometastasis. Wei et al. suggested a 1-year recurrence-free survival (RFS) rate of $14.9 \%$ and 1-year overall survival (OS) rate of $43.1 \%$ in patients who underwent hepatectomy (9). Other studies have reported a 1-year RFS rate of $31.7 \%$ and a median RFS of 4.1 months in patients with PVTT who underwent hepatectomy $(6,10)$. Postoperative adjuvant therapy might also improve patients' survival after hepatectomy.

Angiogenesis is involved in tumor growth and metastasis, and is an important factor in the control of HCC progression. Apatinib is a vascular endothelial growth factor receptor-2 inhibitor that has shown potent antitumor activity and good safety in a variety of solid tumors (11-13), including advanced HCC (14-16). Therefore, it might be a therapeutic option for controlling recurrence after resection of HCC with PVTT. Previous study evaluated an anti-angiogenic therapy, i.e., sorafenib, for patients with relatively low risk of recurrence with a negative result (STORM study). In the present study, we evaluated the efficacy and safety of adjuvant apatinib treatment in patients with HCC and PVTT, an indicator of a high risk of recurrence, after R0 resection, as a novel option for the management of such patients, who otherwise might face a poor prognosis. We present the following article in accordance with the STROBE reporting checklist (available at http://dx.doi.org/10.21037/atm-20-6181).

\section{Methods}

\section{Study design and ethical statement}

This single-center, open-label phase II trial was carried out at Zhongshan Hospital, Fudan University, China. The study was supported by Hengrui Co. Ltd., and registered at ClinicalTrials.gov (\#NCT03261791). The sponsor collaborated with authors on the study design, but the authors collected and analyzed the data, and are accountable for all aspects of the work in ensuring that questions related to the accuracy or integrity of any part of the work are appropriately investigated and resolved. The trial was conducted in accordance with the Declaration of Helsinki (as revised in 2013), and approved by the Ethics Committee of Zhongshan Hospital (No. B2017-065R). Informed consent was given by all individual participants.

\section{Participants}

The eligibility criteria were: (I) $18-70$ years of age; (II) pathologically proven HCC based on the surgically resected specimen; (III) liver resection with curative intention; (IV) PVTT assessed by preoperative imaging or intraoperative findings within 4 weeks of surgery; (V) no preoperative antitumor treatment; (VI) Eastern Cooperative Oncology Group performance status score of 0 or 1; (VII) Child-Pugh score of A or B7; (VIII) adequate organ function, defined as hemoglobin $\geq 90 \mathrm{~g} / \mathrm{L}$, platelets $\geq 80 \times 10^{9} / \mathrm{L}$, total bilirubin $\leq 1.5 \times$ the institutional upper limit of normal (ULN), alanine transaminase (ALT) and aspartate aminotransferase $(\mathrm{AST}) \leq 3 \times \mathrm{ULN}$, and serum creatinine $\leq 1.5 \times \mathrm{ULN}$; and (IX) expected survival $\geq 6$ months.

The key exclusion criteria were: (I) tumor invasion into intrahepatic or extrahepatic bile ducts, combined hepatocellular cholangiocarcinoma, or fibrolamellar HCC; (II) uncontrolled hypertension; and (III) medical history of myocardial ischemia, myocardial infarction, or arrhythmia.

\section{Treatment}

The patients received apatinib monotherapy until they experienced disease recurrence or intolerable toxicity occurred. Apatinib was given orally $(500 \mathrm{mg} /$ day) each day, during a cycle of 4 weeks. Dose adjustments, including interruptions and reductions, were allowed for the management of treatment-related adverse events (AEs). Follow-up imaging examinations (abdominal contrastenhanced magnetic resonance imaging or computed tomography (CT) and chest CT were performed every 2 months or when tumor recurrence was suspected based on elevated serum levels of tumor biomarkers. Complete blood count, liver and renal function tests, serum tumor marker levels, and urine dry chemical analysis were also performed every 2 months during treatment and 1 month 


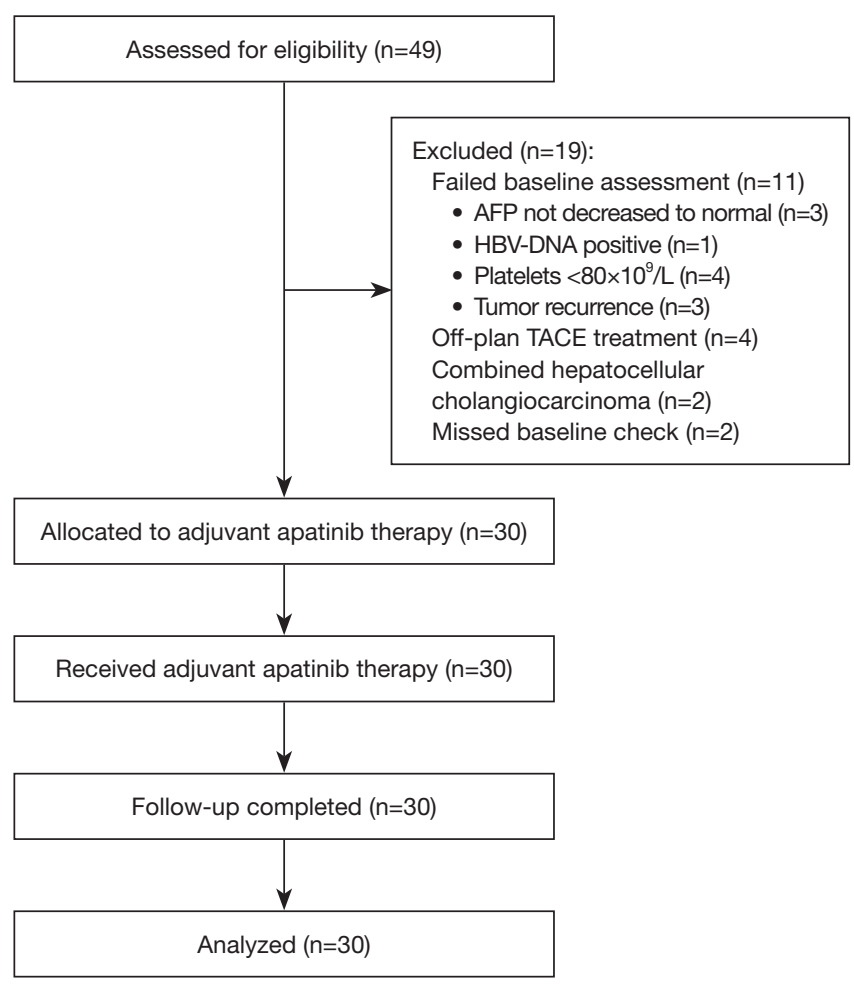

Figure 1 Patient flowchart. AFP, alpha-fetoprotein; HBV, hepatitis $\mathrm{B}$ virus; TACE, transarterial chemoembolization.

after discontinuation of treatment.

\section{Assessment}

The primary endpoint was RFS; tumor recurrence was determined by the investigators. RFS was defined as the time interval from liver resection to the diagnosis of intrahepatic relapse or distant metastasis, or death from any cause. The secondary endpoints included OS and safety. OS was defined as the time from HCC surgery to death from any cause. AEs were recorded and graded according to the National Cancer Institute Common Terminology Criteria for Adverse Events, version 4.0. The investigators assessed the relationship between treatment and AEs. All the AEs reported in this study were treatment-related AEs per investigator assessment.

\section{Statistical analysis}

This was an exploratory study to preliminarily evaluate the efficacy and safety of apatinib as adjuvant treatment in patients who underwent $\mathrm{R} 0$ resection for $\mathrm{HCC}$ and
PVTT; therefore, a small sample size of 30 was planned. The efficacy analyses were conducted on the intent-totreat (ITT) population, which was defined as patients who received at least 1 dose of the study drug. The KaplanMeier product-limit method was used to estimate the median times of RFS and OS in the ITT population. AEs and serious AEs were analyzed in patients who received at least 1 dose of the study drug. Only descriptive statistics were used. Quantitative variables were shown as mean \pm standard deviation or median [interquartile range (IQR)].

\section{Results}

\section{Participant enrollment and baseline characteristics}

Among a total of 49 patients who were screened between August 2017 and December 2018, 30 participants received apatinib (Figure 1). On February 27, 2020, 3 patients were still receiving apatinib treatment at a dosage of $250 \mathrm{mg} / \mathrm{d}$. The baseline characteristics of the 30 participants (ITT population) are presented in Table 1. The median age was 55 years (IQR: 49-62 years), and 25 (83.3\%) patients were male. According to the Liver Cancer Study Group of Japan (LCSGJ) classification of PVTT (17), there were $7(23.3 \%), 11(36.7 \%)$, and $12(40.0 \%)$ participants with $\mathrm{Vp} 1, \mathrm{Vp} 2$, and $\mathrm{Vp} 3$, respectively.

\section{Apatinib treatment}

The median follow-up was 14.3 months (IQR: 12.3-19.3), and the median duration of treatment was 4.8 months (IQR: 2.0-8.8) (Table 2). The median dose of apatinib was $339.7 \mathrm{mg} / \mathrm{d}$ (IQR: $267.7-500 \mathrm{mg} / \mathrm{d}$ ), and the median total drug exposure was $57.2 \mathrm{~g}$ (IQR: 25.8-78.8 g).

\section{Efficacy}

For patients with HCC and PVTT, the median RFS was 7.6 months [95\% confidence interval (CI): 5.7-9.5 months]. The 1 -year RFS rate was $36.1 \%$, and the 1 -year OS was 93.3\% (Table 2, Figure 2).

\section{Safety}

During the treatment period, 29 (96.7\%) patients experienced AEs of any grade, and 14 (46.7\%) experienced grade 3 or 4 AEs. The treatment-related AEs are listed in Table 3. No treatment-related deaths occurred. The most 
Table 1 Characteristics of the participants

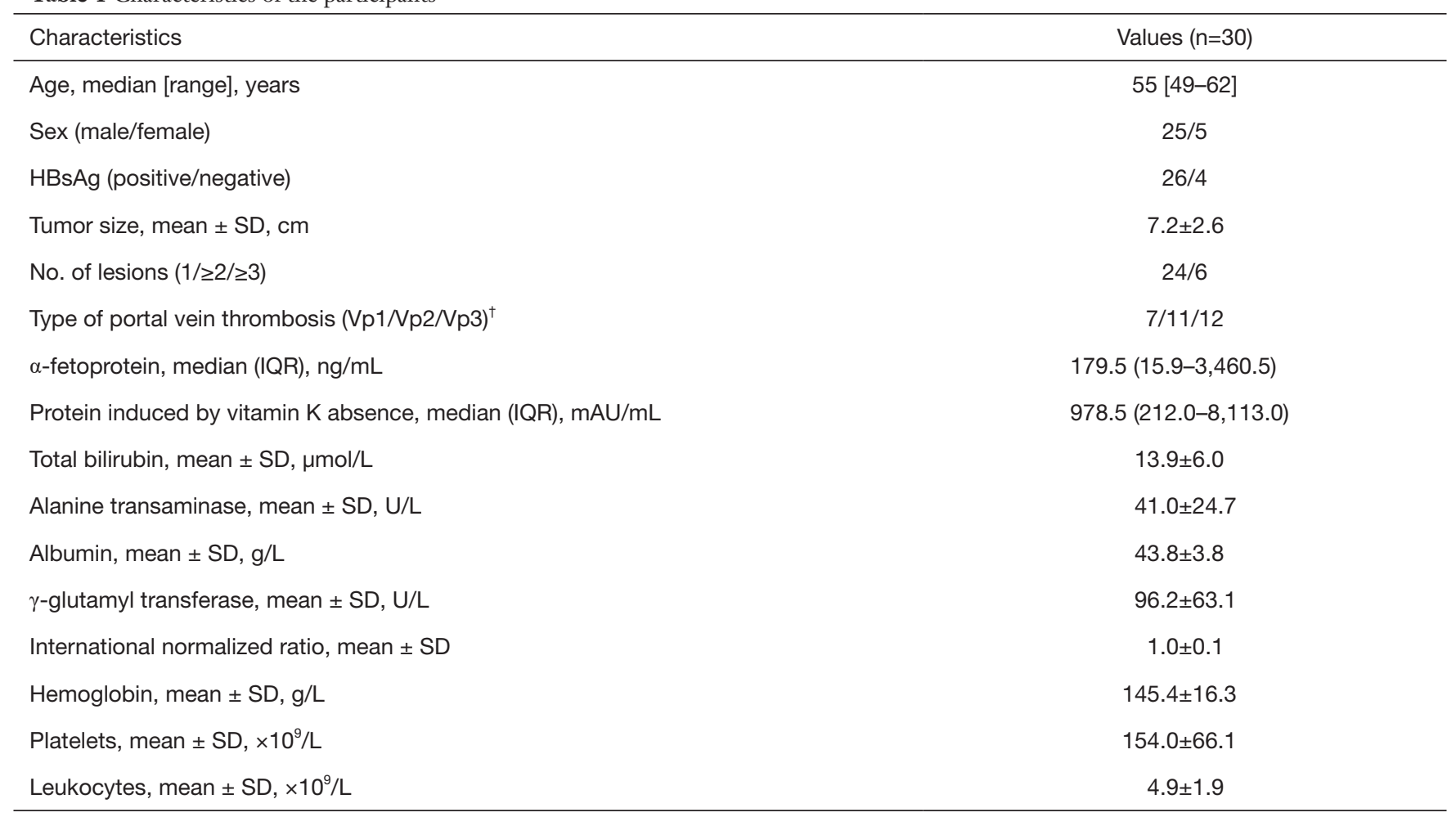

${ }^{\dagger}$, based on the Liver Cancer Study Group of Japan (LCSGJ) classification. IQR, interquartile range; SD, standard deviation.

Table 2 Efficacy evaluation

\begin{tabular}{lc}
\hline Variables & Values \\
\hline Median follow-up (months), median (IQR) & $14.3(12.3-19.3)$ \\
Median duration of treatment (months), & $4.8(2.0-8.8)$ \\
median (IQR) & \\
Median RFS (months) (95\% Cl) & $7.6(5.7-9.5)$ \\
1-year RFS rate (\%) & 36.1 \\
1-year OS rate (\%) & 93.3
\end{tabular}

IQR, interquartile range; LCSGJ, Liver Cancer Study Group of Japan; OS, overall survival PVTT, portal vein tumor thrombosis; RFS, recurrence-free survival.

common treatment-related AEs were thrombocytopenia $(n=16,53.3 \%)$, hand-foot syndrome $(n=14,46.7 \%)$, neutropenia $(n=13,43.3 \%)$, hypertension $(n=9,30.0 \%)$, increased ALT ( $\mathrm{n}=7,23.3 \%)$, and hoarseness ( $\mathrm{n}=7,23.3 \%)$.

Treatment-related grade 3 or 4 AEs included thrombocytopenia $(n=5,16.7 \%)$, neutropenia $(n=4$, $13.3 \%)$, proteinuria $(n=3,10.0 \%)$, hypertension $(n=3$, $10.0 \%)$, increased ALT $(\mathrm{n}=1,3.3 \%)$, increased AST

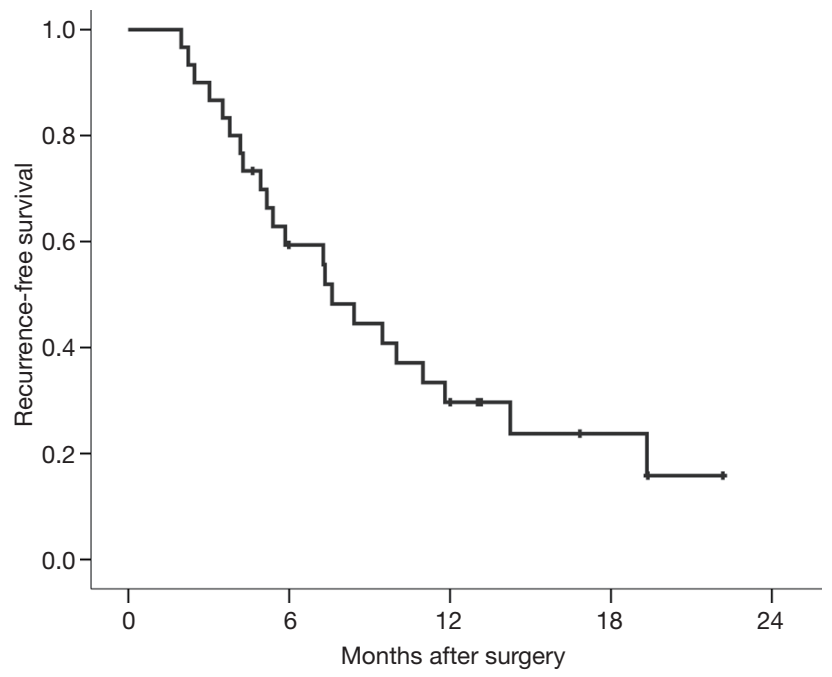

Figure 2 Recurrence-free survival after surgery.

$(\mathrm{n}=1,3.3 \%)$, diarrhea $(\mathrm{n}=1,3.3 \%)$, upper gastrointestinal hemorrhage $(n=1,3.3 \%)$, hoarseness $(n=1,3.3 \%)$, and hand-foot syndrome $(\mathrm{n}=1,3.3 \%)$ (Table 3). The patient with gastrointestinal bleeding and hepatic encephalopathy 
Table 3 Treatment-related adverse events

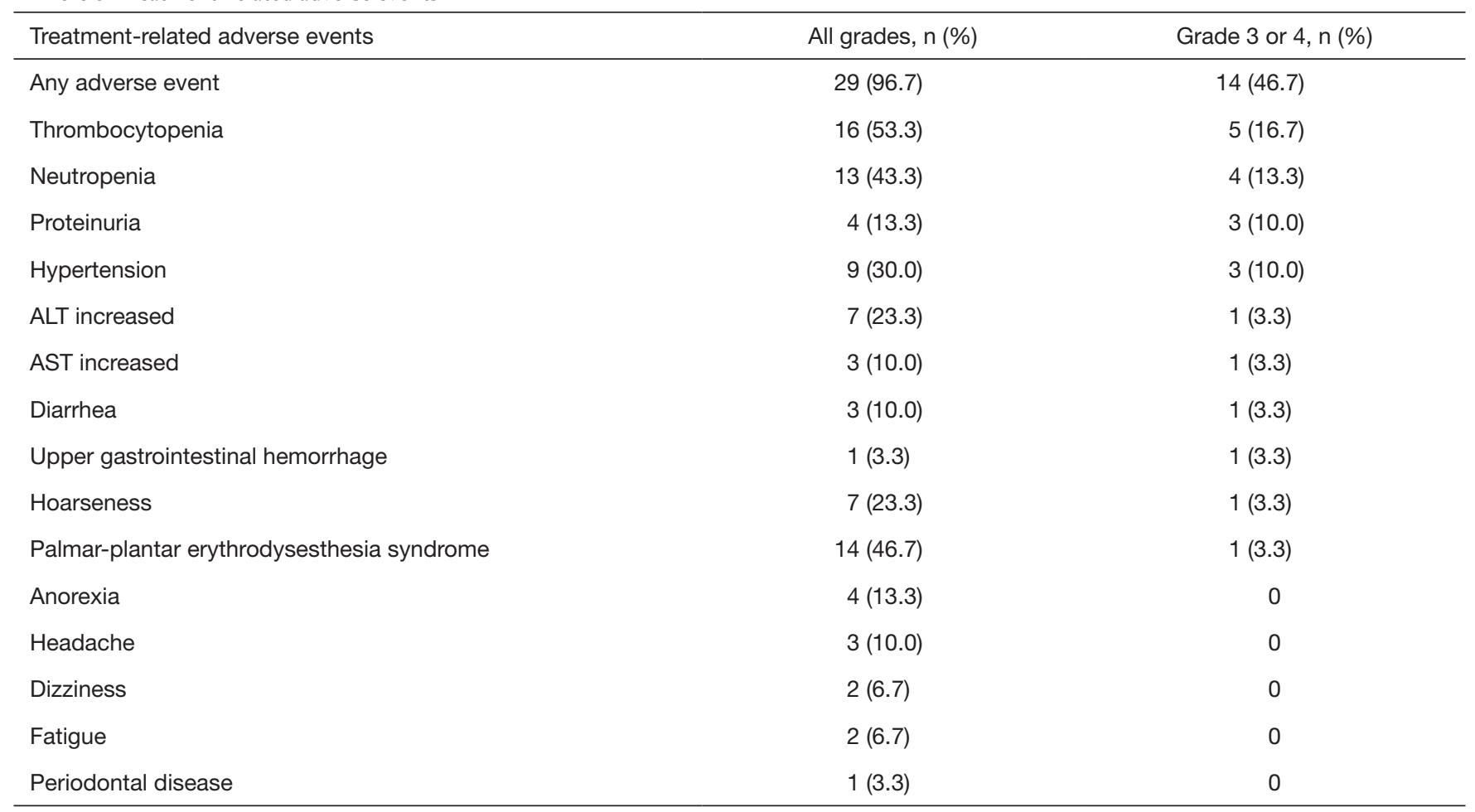

ALT, alanine transaminase; AST, aspartate aminotransferase.

was relieved of symptoms after drug withdrawal and hospitalization.

\section{Discussion}

Patients with HCC and PVTT have a poor prognosis. Because apatinib has been shown to be safe and effective in several solid tumors, including HCC, we conducted the first trial, to the best of our knowledge, evaluating the efficacy and safety of an anti-angiogenesis agent in patients with HCC and PVTT after R0 resection. The results showed that adjuvant apatinib had good treatment efficacy with a tolerable safety profile in these patients.

Previous studies have verified the efficacy of a dose of $500-850 \mathrm{mg} /$ day of apatinib in patients with various solid tumors (11-13,18-26). Moreover, meta-analyses showed that the objective response rate and disease control rate were higher with the 850 and $750 \mathrm{mg} /$ day dosages than with a lower dose (18-26). Most recently, apatinib $750 \mathrm{mg} /$ day showed improved OS in comparison with placebo in a phase III clinical trial when used as second-line therapy in patients with a progressed tumor or those intolerant to previous systemic chemotherapy and/or sorafenib (27). Considering that previous studies have reported $77.4 \%$ treatmentrelated AEs of grade 3 or 4 (28), we chose a lower dosage for apatinib in the adjuvant setting. In this study, apatinib $500 \mathrm{mg} /$ day was well tolerated, and there was $46.7 \%$ treatment-related AEs of grade 3 or 4 .

Recent studies that included Eastern Asian patients suggested that surgical resection could be a treatment option for patients with Barcelona Clinic Liver Cancer (BCLC) C stage HCC, especially those with type I or II PVTT. Peng et al. (29) found a 1-year RFS and OS of $63.0 \%$ and $81.5 \%$, respectively, in HCC patients with type I PVTT, and $20.9 \%$ and $46.3 \%$, respectively, for type II PVTT among those who underwent hepatic resection for HCC. Kokudo et al. (30) examined patients with R1 or R2 resection, reporting a favorable long-term survival after resection with median RFS and OS for $\mathrm{Vp} 1, \mathrm{Vp} 2$, and $\mathrm{Vp} 3$ of 1.23 and 4.13 years, 0.82 and 2.49 years, and 0.56 and 1.58 years, respectively. Moreover, Zhang et al. (31) showed that for patients with type I or II PVTT and R0 resection (type I PVTT represented $34-48.7 \%$ of the patients), the median RFS was 4.9-8.1 months, and median OS was 9.3-10.4 months. In a group of patients with predominantly type I PVTT ( $>90 \%)$, the median RFS and OS were 11.0 
and 12.4 months, respectively. Therefore, the extent of PVTT is associated with survival in patients with HCC. Patients with more extensive PVTT possibly have a larger residual lesion after surgery, and in those cases, adjuvant apatinib might be an option.

In the present study, the 1-year (36.1\%) and median RFS (7.6 months), and 1-year OS (93.3\%) were higher than in a previous study that recruited similar participants randomized to surgery (1-year OS, $43.1 \%$ ) versus surgery plus radiation therapy (1-year OS, 75.2\%) (9). These differing results could be partly explained by that the patients recruited in the present study had a better performance status than previous study, but also by the use of apatinib, which needs to be examined in a controlled trial. Nevertheless, the OS was substantially longer than the 8.9 months and 5.6 months (HCC with macrovascular invasion and/ or extrahepatic spread) reported by the SHARP (32) and Asia-Pacific (33) trials, respectively, for sorafenib monotherapy. In addition, a small-series study reported that adjuvant sorafenib after HCC resection improved the time to progression and OS compared with surgery alone in patients with HCC and PVTT (34). Patients with adjuvant sorafenib therapy had an improved time to progression (29 vs. 22 months, $\mathrm{P}=0.041$ ) and an improved OS (37 vs. 30 months, $\mathrm{P}=0.01$ ).

Anti-angiogenesis targeted therapy has been shown to delay wound healing (35), so in the present trial, apatinib was started 4-6 weeks after surgery to ensure safety. The occurrence of grade 3 or $4 \mathrm{AE}$ was, in general, comparable to that in the STORM trial conducted with patients who received sorafenib after HCC resection (i.e., $50 \%$ for grade 3 AEs and 2\% for grade 4) (36). The most common apatinib-related AEs were hypertension and proteinuria, consistent with the known toxicity profile $(11,14,15,18-20$, 22-24,26). No new toxicity signals were identified.

There are some limitations to the present study that need to be pointed out. This was a single-arm exploratory trial without a control group or randomization and the sample size is small. Thus, the results are not conclusive. In future study, we should introduce a control group with standard treatment in clinical practice (e.g., TACE). Nevertheless, our results revealed a high RFS and 1-year OS, suggesting that further studies on apatinib for HCC and PVTT might be warranted.

\section{Conclusions}

Apatinib was well tolerated after R0 resection for HCC with PVTT. The median RFS in this group was improved compared with previous reports. However, further, larger sample size trials, which will include a control group, should be performed to confirm these findings. Based on this study and another study carried out in our hospital (adjuvant therapy with apatinib plus SHR1210, clinicaltrial.gov identifier NCT03722875), a phase III randomized placebocontrolled clinical trial evaluating apatinib plus SHR1210 as adjuvant therapy is in plan.

\section{Acknowledgments}

The authors thank the patients and their families, and thank Mei-Ling Li and Jin-Jin Zhu for assisting with the data collection.

Funding: This work was supported by Jiangsu Hengrui Medicine Co., Ltd.

\section{Footnote}

Reporting Checklist: The authors have completed the STROBE reporting checklist. Available at http://dx.doi. org/10.21037/atm-20-6181

Data Sharing Statement: Available at http://dx.doi. org/10.21037/atm-20-6181

Conflicts of Interest: All authors have completed the ICMJE uniform disclosure form (available at http://dx.doi. org/10.21037/atm-20-6181). HCS reports personal fees from Bayer, personal fees from Eisai, personal fees from MSD, personal fees from Hengrui Co., Ltd., personal fees from Innovent Biologics, outside the submitted work; XDZ reports personal fees from Eisai, personal fees from MSD, personal fees from Hengrui Co. Ltd., outside the submitted work. The other authors have no conflicts of interest to declare.

Ethical Statement: The authors are accountable for all aspects of the work in ensuring that questions related to the accuracy or integrity of any part of the work are appropriately investigated and resolved. The trial was conducted in accordance with the Declaration of Helsinki (as revised in 2013). The study was approved by the ethics committee of Zhongshan Hospital (No. B2017-065R) and informed consent was given by all individual participants.

Open Access Statement: This is an Open Access article 
distributed in accordance with the Creative Commons Attribution-NonCommercial-NoDerivs 4.0 International License (CC BY-NC-ND 4.0), which permits the noncommercial replication and distribution of the article with the strict proviso that no changes or edits are made and the original work is properly cited (including links to both the formal publication through the relevant DOI and the license). See: https://creativecommons.org/licenses/by-nc-nd/4.0/.

\section{References}

1. Bray F, Ferlay J, Soerjomataram I, et al. Global cancer statistics 2018: GLOBOCAN estimates of incidence and mortality worldwide for 36 cancers in 185 countries. CA Cancer J Clin 2018;68:394-424.

2. Cheung TT. Management of hepatocellular carcinoma: from bench to bedside and beyond. Transl Gastroenterol Hepatol 2019;4:54.

3. Park JW, Chen M, Colombo M, et al. Global patterns of hepatocellular carcinoma management from diagnosis to death: the BRIDGE Study. Liver Int 2015;35:2155-66.

4. A new prognostic system for hepatocellular carcinoma: a retrospective study of 435 patients: the Cancer of the Liver Italian Program (CLIP) investigators. Hepatology 1998;28:751-5.

5. Holzwanger DJ, Madoff DC. Role of interventional radiology in the management of hepatocellular carcinoma: current status. Chin Clin Oncol 2018;7:49.

6. Sun JX, Shi J, Li N, et al. Portal vein tumor thrombus is a bottleneck in the treatment of hepatocellular carcinoma. Cancer Biol Med 2016;13:452-8.

7. Zhou J, Sun HC, Wang Z, et al. Guidelines for Diagnosis and Treatment of Primary Liver Cancer in China (2017 Edition). Liver Cancer 2018;7:235-60.

8. Marrero JA, Kulik LM, Sirlin CB, et al. Diagnosis, Staging, and Management of Hepatocellular Carcinoma: 2018 Practice Guidance by the American Association for the Study of Liver Diseases. Hepatology 2018;68:723-50.

9. Wei X, Jiang Y, Zhang X, et al. Neoadjuvant ThreeDimensional Conformal Radiotherapy for Resectable Hepatocellular Carcinoma With Portal Vein Tumor Thrombus: A Randomized, Open-Label, Multicenter Controlled Study. J Clin Oncol 2019;37:2141-51.

10. Sun J, Zhang T, Wang J, et al. Biologically effective dose (BED) of stereotactic body radiation therapy (SBRT) was an important factor of therapeutic efficacy in patients with hepatocellular carcinoma $(</=5 \mathrm{~cm})$. BMC Cancer 2019;19:846.
11. Scott LJ. Apatinib: A Review in Advanced Gastric Cancer and Other Advanced Cancers. Drugs 2018;78:747-58.

12. Liu G, Wang C, He Y, et al. Application effect of apatinib in patients with failure of standard treatment for advanced malignant tumours. BMC Pharmacol Toxicol 2019;20:61.

13. Yang D, Dai R, Zhang Q, et al. Apatinib for heavily treated patients with non-small cell lung cancer: Report of a case series and literature review. Saudi J Biol Sci 2018;25:888-94.

14. Zhang Y, Fan W, Wang Y, et al. Apatinib for Patients With Sorafenib-Refractory Advanced Hepatitis B Virus Related Hepatocellular Carcinoma: Results of a Pilot Study. Cancer Control 2019;26:1073274819872216.

15. Kong Y, Sun L, Hou Z, et al. Apatinib is effective for treatment of advanced hepatocellular carcinoma. Oncotarget 2017;8:105596-605.

16. Qin S. Apatinib in Chinese patients with advanced hepatocellular carcinoma: A phase II randomized, openlabel trial. J Clin Oncol 2014;32:abstr 4019.

17. Kudo M, Izumi N, Kokudo N, et al. Management of hepatocellular carcinoma in Japan: Consensus-Based Clinical Practice Guidelines proposed by the Japan Society of Hepatology (JSH) 2010 updated version. Dig Dis 2011;29:339-64.

18. Chen J, Wang J. Efficacy and safety assessment of apatinib in patients with advanced gastric cancer: a meta-analysis. Onco Targets Ther 2018;11:4149-58.

19. Cheng H, Sun A, Guo Q, et al. Efficacy and safety of apatinib combined with chemotherapy for the treatment of advanced gastric cancer in the Chinese population: a systematic review and meta-analysis. Drug Des Devel Ther 2018;12:2173-83.

20. Liu Y, Zhou C, Zhang K, et al. The combination of apatinib and S-1 for the treatment of advanced gastric cancer in China: A meta-analysis of randomized controlled trials. Medicine (Baltimore) 2018;97:e13259.

21. Peng L, Ye X, Hong Y, et al. Treatment-related toxicities of apatinib in solid tumors: a meta-analysis. Oncotarget 2018;9:32262-70.

22. Sun D, Hou H, Zhang C, et al. The efficacy and safety of apatinib for refractory malignancies: a review and metaanalysis. Onco Targets Ther 2018;11:6539-54.

23. Wei Y, Liu J, Yan M, et al. Effectiveness and Safety of Combination Therapy of Transarterial Chemoembolization and Apatinib for Unresectable Hepatocellular Carcinoma in the Chinese Population: A Meta-Analysis. Chemotherapy 2019;64:94-104.

24. Xue JM, Astere M, Zhong MX, et al. Efficacy and safety of apatinib treatment for gastric cancer, hepatocellular 
carcinoma and non-small cell lung cancer: a meta-analysis. Onco Targets Ther 2018;11:6119-28.

25. Yu GC, Yang J, Ye B, et al. Apatinib in the treatment of advanced non-small-cell lung cancer: A meta-analysis. Math Biosci Eng 2019;16:7659-70.

26. Zhao S, Zhang T, Dou W, et al. A comparison of transcatheter arterial chemoembolization used with and without apatinib for intermediate- to advanced-stage hepatocellular carcinoma: a systematic review and metaanalysis. Ann Transl Med 2020;8:542.

27. Li Q, Qin S, Gu S, et al. Apatinib as second-line therapy in Chinese patients with advanced hepatocellular carcinoma: A randomized, placebo-controlled, double-blind, phase III study. J Clin Oncol 2020;38:abstr 4507.

28. Zhu XD, Li KS, Sun HC. Adjuvant therapies after curative treatments for hepatocellular carcinoma: Current status and prospects. Genes Dis 2020;7:359-69.

29. Peng ZW, Guo RP, Zhang YJ, et al. Hepatic resection versus transcatheter arterial chemoembolization for the treatment of hepatocellular carcinoma with portal vein tumor thrombus. Cancer 2012;118:4725-36.

30. Kokudo T, Hasegawa K, Matsuyama Y, et al. Survival benefit of liver resection for hepatocellular carcinoma associated with portal vein invasion. J Hepatol 2016;65:938-43.

31. Zhang YF, Shang H, Zeng XL, et al. Postoperative

Cite this article as: Sun HC, Zhu XD, Zhou J, Gao Q, Shi YH, Ding ZB, Huang C, Qiu SJ, Ren N, Shi GM, Sun J, Ye QH, Huang XW, Yang XR, Fan J. Adjuvant apatinib treatment after resection of hepatocellular carcinoma with portal vein tumor thrombosis: a phase II trial. Ann Transl Med 2020;8(20):1301. doi: 10.21037/atm-20-6181 adjuvant chemo (embolization) therapy for hepatocellular carcinoma with portal vein tumor thrombosis. Onco Targets Ther 2018;11:5407-17.

32. Sherman M, Mazzaferro V, Amadori D, et al. Efficacy and safety of sorafenib in patients with advanced hepatocellular carcinoma and vascular invasion or extrahepatic spread: A subanalysis from the SHARP trial. J Clin Oncol 2008;26:abstr 4584.

33. Cheng AL, Guan Z, Chen Z, et al. Efficacy and safety of sorafenib in patients with advanced hepatocellular carcinoma according to baseline status: subset analyses of the phase III Sorafenib Asia-Pacific trial. Eur J Cancer 2012;48:1452-65.

34. Li J, Hou Y, Cai XB, et al. Sorafenib after resection improves the outcome of BCLC stage C hepatocellular carcinoma. World J Gastroenterol 2016;22:4034-40.

35. Bodnar RJ. Anti-Angiogenic Drugs: Involvement in Cutaneous Side Effects and Wound-Healing Complication. Adv Wound Care (New Rochelle) 2014;3:635-46.

36. Bruix J, Takayama T, Mazzaferro V, et al. Adjuvant sorafenib for hepatocellular carcinoma after resection or ablation (STORM): a phase 3, randomised, double-blind, placebo-controlled trial. Lancet Oncol 2015;16:1344-54.

(English Language Editor: K. Brown) 